\title{
CAMPOUT: A Control Architecture for Tightly Coupled Coordination of Multirobot Systems for Planetary Surface Exploration
}

\author{
Terry Huntsberger, Paolo Pirjanian, Ashitey Trebi-Ollennu, Hari Das Nayar, Hrand Aghazarian, Anthony J. Ganino,
} Mike Garrett, Associate Member, IEEE, Sanjay S. Joshi, and Paul S. Schenker, Member, IEEE

\begin{abstract}
Exploration of high risk terrain areas such as cliff faces and site construction operations by autonomous robotic systems on Mars requires a control architecture that is able to autonomously adapt to uncertainties in knowledge of the environment. We report on the development of a software/hardware framework for cooperating multiple robots performing such tightly coordinated tasks. This work builds on our earlier research into autonomous planetary rovers and robot arms. Here, we seek to closely coordinate the mobility and manipulation of multiple robots to perform examples of a cliff traverse for science data acquisition, and site construction operations including grasping, hoisting, and transport of extended objects such as large array sensors over natural, unpredictable terrain. In support of this work we have developed an enabling distributed control architecture called control architecture for multirobot planetary outposts (CAMPOUT) wherein integrated multirobot mobility and control mechanisms are derived as group compositions and coordination of more basic behaviors under a task-level multiagent planner. CAMPOUT includes the necessary group behaviors and communication mechanisms for coordinated/cooperative control of heterogeneous robotic platforms. In this paper, we describe CAMPOUT, and its application to ongoing physical experiments with multirobot systems at the Jet Propulsion Laboratory in Pasadena, CA, for exploration of cliff faces and deployment of extended payloads.
\end{abstract}

Index Terms-Distributed control architecture, multiple mobile robots, robot outposts, tight coordination.

\section{INTRODUCTION}

$\mathbf{F}$ UTURE robotic exploration of Mars will likely entail cooperative activity of multiple robots such as those described in [1] and examples shown in Fig. 1. Fig. 1(a) shows the possible water outflow zones at the tops of cliffs on Mars, and a robotic outpost concept drawing in Fig. 1(d), along with JPL

Manuscript received July 15, 2002; revised April 25, 2003. This work was supported under Contract by the National Aeronautics and Space Administration and carried out by the Jet Propulsion Laboratory, California Institute of Technology. This paper was presented in part at the IEEE International Conference on Robotics and Automation [53], [54]. This paper was recommended by Guest Editor H. Zhang.

T. Huntsberger, A. Trebi-Ollennu, H. Aghazarian, A. J. Ganino, M. Garrett and P. S. Schenker are with the Jet Propulsion Laboratory, Pasadena, CA 91109 USA (e-mail: Terry.Huntsberger@jpl.nasa.gov).

P. Pirjanian is with Evolution Robotics, Inc., Pasadena, CA 91103 USA (e-mail: paolo@evolution.com).

H. D. Nayar is with OphirTech, Altadena, CA 91003 USA (e-mail: hari@ophirtech.com).

S. S. Joshi is with the Department of Mechanical and Aeronautical Engineering, University of California, Davis, 95616 CA USA (e-mail: maejoshi@ucdavis.edu).

Digital Object Identifier 10.1109/TSMCA.2003.817398

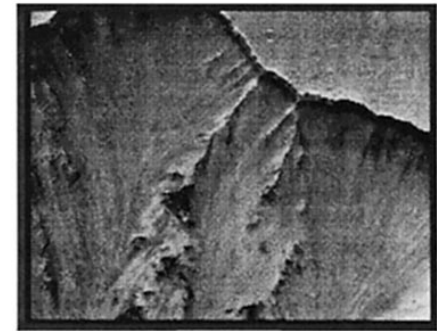

(a)

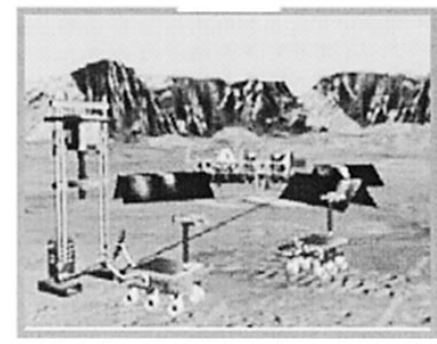

(c)

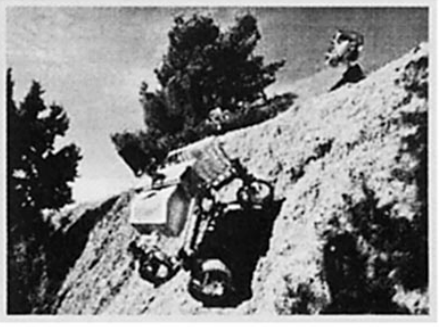

(b)

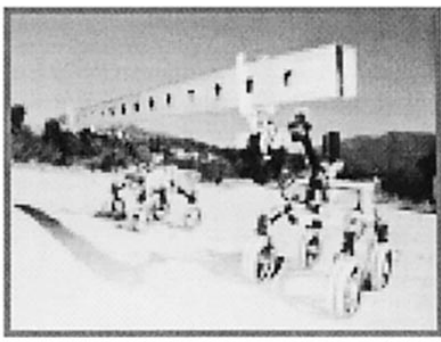

(d)
Fig. 1. Mars exploration environments and JPL technology prototypes. (a) Mars cliff-face with evidence of water outflows. (b) Technology concept cliff-bot ensemble. (c) Artist concept of Mars robotic outpost. (d) Technology concept robotic work crew.

technology prototype systems for these environments. These missions include the use of multiple, heterogeneous, mobile robotic platforms for surface exploration and infrastructure deployment. A high degree of autonomy is necessary, since the delays of the long communication path to Mars limit the amount of teleoperation that is possible. The cooperating robots will work as "teams" of coordinated intelligent agents, carrying out site preparations, site maintenance functions, and remote science investigations, eventually in partnership with human cohabitants of such planetary outposts. We have developed a control architecture for such teams, called control architecture for multirobot planetary outposts (CAMPOUT). We have validated this architecture through the development and field experimentation with such robotic system concepts, building on prior JPL work in autonomous planetary rovers and robots, e.g., our recent development of the MarsArm, LSR, SRR, and FIDO platforms [2]-[6].

The main motivation for our work, taken from previous studies of robotic requirements for Mars robotic outposts [43], is that increased levels of autonomy and more generalized payload handling capabilities than have been reported to date will 
be needed for habitat construction and surface infrastructure support on planetary surfaces. The applications challenge is further exacerbated by the unstructured nature of the planetary surface environment (often unpredictable with respect to both character of perceptual artifacts and poorly modeled nature of vehicle-surface interactions), and the extended duration and changing goals/priorities of such missions. A generalized behavior-based control appears to offer a practical level of flexibility, autonomy, and computational economy [42], [52] for preliminary design of such space-targeted technologies and systems.

Our research focuses on multirobot cooperation for tasks that inherently require tight coordination under strict physical constraints. Such tightly coupled coordination tasks are characterized by constraints imposed on the activities of one robot as a function of the state of others. Most work on multirobot systems has to date been limited to tasks such as collective estimation [8], [9] (e.g., mapping and localization) cooperative foraging [10]-[13], [46], and cooperative box pushing [14]-[16], where tight coordination of the activities of the robots is not required. Collective estimation and foraging tasks can be performed independently by each robot and usually do not require a tight coordination of activities. Cooperative box pushing requires tighter cooperation but can be accomplished by turn-taking schemes where each robot can alternate in pushing one end of the box toward a goal. But since the box rests on a surface, the activities of the robots do not need to be closely orchestrated simultaneously.

Recently, robotics researchers have investigated transportation of large extended objects using autonomous cooperating or coordinated multiple robots (wherein the latter term, coordinated, infers tight coupling of the physical platforms' kinematics and dynamical parameters) [17]-[31], [57], [61]. Compliant control for multiple mobile robots is very different from that of a single mobile robot. First, the compliance frame is implicitly time varying, and second, the environment is not static because the contact occurs or is maintained while all robots are in motion. In general, we note that many approaches reported for cooperative robot motion do not generalize; they may not consider activity within a natural terrain, versus an idealized environment (lab floor), and/or fail to maintain an explicit continuous closed loop coordination of joint robot activities under physical constraints (rather, using time-sequenced, iterative actions of the independent robots to partially address global task constraints). Activities may be cooperative in a spatial sense, but not necessarily coordinated below a strategic level as to platform kinematics and inertial/dynamical interactions.

The tasks of cooperative mobile object grasping, manipulation, and handling [17]-[31], [57], [61] (e.g., lifting and carrying, not pushing, a piano up the stairs), or tethered cliff descent [54], [55] require tight and simultaneous (vs. turn-taking) coordination of each robot in order to maintain grip of the object while manipulating/handling it or stability of the robot on the cliff face. State of the art is currently limited to indoor lab demonstrations on a level floor (with the exceptions of [22], [30], [31]) often using omni-directional mobile manipulators with multiple degrees of freedom.
Vinay et al. [29] presented simulation results of two mobile robots transporting a long object using Lagrange techniques to develop a state space model for two wheeled mobile robots compliantly coupled to a common payload. Hisashi et al. [22] also presented simulation and experimental results of two cooperative mobile manipulators transporting a payload on uneven ground. In the reported experiments, the robots and the payload consisted of three moving tables driven by ball screws. Simple joint position control laws are employed to accomplish compliant control between the mobile manipulators without the need for explicit communication. Hara et al. [21] presented a cooperative transportation control scheme for two quadruped robots transporting a long payload. A decentralized control scheme is developed based on a "leader-follower." Wang et al. [62] presented a system called behavior-based multiple robot system with host for object manipulation (BeRoSH) in which the collection of homogeneous robots operate under the control of a managing robot. The managing robot generates goals for the other team members, who then use a form closure concept to build their local goals and behavior mechanism.

Khatib et al. [17], [18] proposed a somewhat more general decentralized cooperative control algorithm for multiple mobile manipulators using an augmented object and a virtual linkage model. The augmented object is used to describe the system's closed chain dynamics. The virtual link model is used to characterize and synthesis control laws for internal forces in a multiarm systems. However, the algorithm requires an explicit and not always realistically achieved communication between the platforms.

Robot control architectures can be broadly characterized as deliberative (based on planning), reactive (tight coupling between sensing and actuation), or a hybrid blend of the two [32]. Behavior-based systems approach autonomy from the standpoint of collections of behaviors [60]. Such systems were introduced by Brooks [33] and further extended by Arkin [11], Parker [16], and Mataric [13] among others. The autonomous robot architecture (AuRA) [36], Atlantis [41], and the plannerreactor architecture [59] are some examples of hybrid control systems. The wide range of possible behaviors that are needed for a planetary rover obviates the need for an action selection mechanism (ASM) to provide the correct behavior for any given situation. Comprehensive reviews of behavior coordination (or action selection) mechanisms can be found in Arkin [60] and Pirjanian [39]. Recent work of Pirjanian and Mataric [39], [40] using multiple objective decision making (MODM) provides formal tools for generating strategies that can guarantee an appropriate trade-off between the optimal solutions, which are possibly not reachable in a planetary surface environment, and Pareto optimal or satisficing ("good enough") solutions.

Behavior coordination in multirobot systems has received relatively little attention. One approach proposed in [14] uses inhibition and suppression across a network of heterogeneous robots augmented with motivational behaviors that can trigger behavior invocation based on some internal parameters that measure progress. A similar approach was proposed in the AYLLU architecture [56], which uses port arbitration as the main mechanism for multirobot behavior coordination. Both of these approaches can be viewed as the extension of subsump- 
tive-style arbitration to multirobot coordination. Recently, work in progress is investigating the extension of the 3T architecture to multirobot coordination [51]. The above approaches as well as most multirobot architectures including ACTRESS, GOFER, SWARM, [48]-[50] are mostly limited to execution of tasks that are either independent/parallel or loosely coupled, turn-taking tasks. A comprehensive review can be found in [45].

In this paper, we describe the underlying basis and implementation of CAMPOUT [30], [31], and physical experimentation to date with two rovers grasping, hoisting, and carrying a model payload over natural terrain and a cliff descent involving three agents. Such tasks involve a collection of heterogeneous robotic platforms, under frequently varying control and communications protocols due to the wide range of tasks (some unforeseeable) that they will be required to do. The control architecture must therefore not be a "point design," but rather, extensible and expandable. Tasks may include not only site preparation and maintenance functions, but also support of science goals (instrument deployments, sample transport, in-field rendezvous, etc.). Sections II and III give an overview of the control architecture. Section IV gives the details of the experimental studies, and we conclude with Section V.

\section{CAMPOUT}

CAMPOUT (shown in Fig. 2) is a distributed control architecture based on a multiagent behavior-based methodology, wherein higher-level functionality is composed by coordination of more basic behaviors under the downward task decomposition of a multiagent planner. CAMPOUT provides the infrastructure, tools, and guidelines that consolidate a number of diverse techniques to allow the efficient use and integration of these components for meaningful interaction and operation. This is facilitated through a few elementary architectural mechanisms for behavior representation, behavior composition, and behavior and group coordination, and the interfaces between these. These mechanisms and a framework with guidelines for describing systems define the core of CAMPOUT. CAMPOUT is thus extensible and scales freely with regard to behavioral mechanisms and protocols it can host and fuse, remappable inter-robot communications it can support, and the overall ability to functionally integrate heterogeneous, multipurpose platforms.

\section{A. Behavior Representation}

In our architectural methodology we formalize a behavior, $b$, as a mapping, $b: P^{*} \times X \rightarrow[0 ; 1]$, that relates each percept sequence $p \in P^{*}$ and action $x \in X$ pair, $(p, x)$, to a preference value that reflects the action's desirability. The percept describes possible (processed or raw) sensory input and the N-dimensional action space is defined to be a finite set of alternative actions. The described mapping assigns to each action $x \in X$ a continuous valued preference, where the most desired actions are assigned 1, and undesired actions are assigned 0 from that behaviors point of view. In CAMPOUT this representation is implemented using finite state machines (format used for NASA

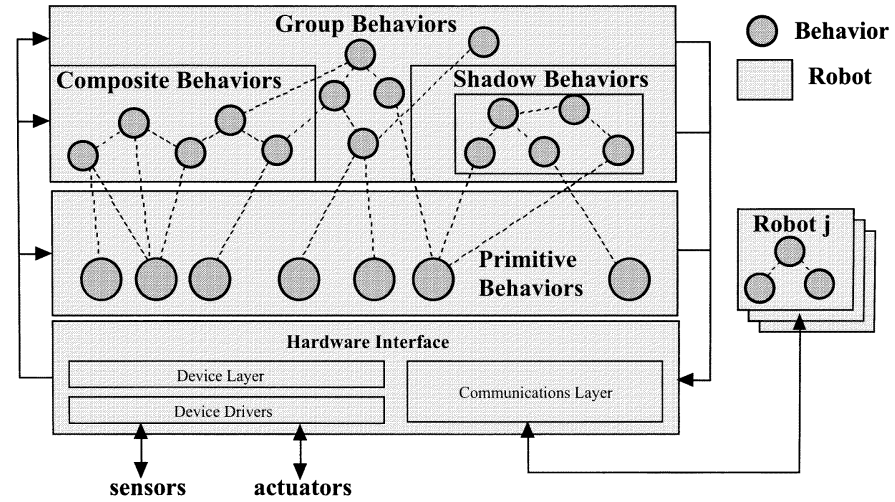

Fig. 2. Schematic overview of CAMPOUT and its hierarchical organization in terms of primitive behaviors, composite behaviors built from primitive behaviors and group behaviors that are composed from coordination of behaviors across multiple robots. Implicit communication is enabled through the shadow behaviors. Each robot runs an instance of this architecture and coordinates activities through group behaviors, which is facilitated through the communications behaviors.

rover missions) in order to maintain access to internal state information.

\section{B. Behavior Composition}

Behavior composition refers to the mechanisms used for building higher-level behaviors by combining lower-level ones. A major issue in the design of behavior-based control systems is the formulation of effective mechanisms for coordination of the behaviors' activities into strategies for rational and coherent behavior. Behavior coordination mechanisms (BCMs) manage the activities of lower-level behaviors within the context of a high-level behavior's task and objectives. For a detailed overview, discussion, and comparison of behavior coordination mechanisms see [32].

\section{Behavior Coordination Mechanisms}

If behaviors are viewed as operands, then BCMs are the operators used to combine behaviors into higher-level behaviors. In this section we describe the BCMs that are used in CAMPOUT for behavior composition. BCMs can be divided into two complementary classes: arbitration and command fusion.

Arbitration mechanisms select one behavior, from a group of competing ones, and give it ultimate control of the system (the robot) until the next selection cycle. This approach is suitable for arbitrating between the set of active behaviors in accord with the system's changing objectives and requirements under varying conditions. It can focus the use of scarce system resources (sensory, computational, etc.) on tasks that are considered to be relevant. CAMPOUT implements priority-based arbitration, where behaviors with higher priorities are allowed to suppress the output of behaviors with lower priorities; and state-based arbitration which is based on the discrete event systems (DES) formalism [34], and is suitable for behavior sequencing.

Command fusion mechanisms combine recommendations from multiple behaviors to form a control action that represents their consensus. This approach provides for a coordination scheme that allows all behaviors to simultaneously contribute 
to the control of the system in a cooperative rather than a competitive manner, which makes them suitable for tightly coupled tasks that require spatio-temporal coordination of activities. CAMPOUT uses the following command fusion mechanisms:

- voting techniques that interpret the output of each behavior as votes for or against possible actions and the action with the maximum weighted sum of votes is selected (DAMN-style [35] voting algorithm based on BISMARC [44], [47], [58]);

- fuzzy command fusion mechanisms (see [37], [38]) that use fuzzy logic and inference to formalize the action selection processes;

- multiple objective behavior fusion mechanisms that select an action with the best trade-off between the task objectives and which satisfies the behavioral objectives as much as possible based on multiple objective decision theory [39].

\section{Group Coordination}

In order to cooperate and collectively contribute to a common task, the robots must cooperate and coordinate their activities. Behavior coordination is basically concerned with resolving or managing conflicts between mutually exclusive alternatives and between behavioral objectives. Group coordination in CAMPOUT is treated as the coordination of multiple distributed behaviors, across a network of robots, where more than one decision maker is present. In order to support this view, BCMs are extended to support multi-robot coordination, as was done by Pirjanian and Mataric [52].

\section{E. Communication Behaviors}

In order to facilitate a group of robots to coordinate their activities and cooperate toward the accomplishment of a common task, they may be required to communicate in order to share resources (e.g., sensors or actuators), exchange information (e.g., state, percepts), synchronize their activities, etc. The behaviors and hence the robots can communicate implicitly by interaction through the environment or using sensory feedback, or explicitly through direct communication. Communication is not necessarily limited to explicit exchange of information via some sort of a data link, but can also include visual, auditory, tactile, and other types of communication. For instance a robot can determine the relative position of another robot using cameras. Alternatively, the other robot could explicitly transmit its position within a global coordinate system.

Currently, CAMPOUT uses UNIX-style sockets for communication. The communications facilities consist of the following core functions.

- Synchronization: Two main functions signal (destination, sig) and wait (source, sig) are used to send and wait for a signal to and from a given robot. This pair constitutes the facilities for synchronizing the activities of robots and/or behaviors.

- Data exchange: SendEvent (destination, event) and GetEvent (source, event) are used to send and receive an event structure to and from a particular robot. The event structure can contain arbitrary data packages as contracted between the sender (source) and receiver (destination). For instance, it can be used to transmit a percept or raw sensor data from one robot to the other etc. For example, robot 2 will be able to have a behavior that is being fed by the position of robot 1 (to, e.g., follow it).

- Behavior exchange: SendObjective (destination, objective) and GetObjective (source, objective) are used to send and receive objective functions (multivalued behavior outputs) to and from a robot. Using these functions one can form a network of behaviors across a distributed group of robots.

\section{COORDINATED OBJECT TRANSPORTATION}

There are numerous challenges in a prototypical task such as an autonomous PV tent deployment on a planetary surface [7], including the cooperative manipulative acquisition of extended objects from a container storage depot, the cooperative transport of such a container to the power array construction site, and the physical deployment of the container into the array. We will use this task as an example in order to illustrate the types of behaviors needed, behavior composition mechanism, and the implementation under CAMPOUT. The main robotics requirements for this task include coordinated grasping and navigation over open terrain by two or more cooperating robots. Navigation over unconstrained terrain will prove to be a significant challenge, especially with Mars-like rovers with severe holonomic constraints. In addition, there must be accurate localization of the robots as the PV tent containers are unloaded from a container storage unit (CSU) and delivered to the site, since damage to the solar tents could otherwise occur. The coordinated transport task in open, uneven terrain requires a tightly-coupled, close coordination of the activities of the two robots. This is accomplished by some 20 behaviors, organized in a hierarchy as shown in Fig. 3.

There are two main group behaviors used in CAMPOUT for this task. Assume formation and approach target. The assume formation group behavior is used to turn the formation to face the deployment target area and is invoked each time the heading error relative to the target is larger than a preset threshold $\left(5^{\circ}\right.$ in our experimental studies). The approach target group behavior uses a visual target finding algorithm based on color-segmentation to localize the rovers for heading adjustments during the traverse step in the sequence.

Key to these group behaviors is the notion of compliance by implicit communication through the shared container (position of other rover can be inferred using shadow behaviors tied to sensing of the payload carried by the two rovers) and explicit communication through communication behaviors for distributed resource sharing. The compliance behaviors assure safe handling of the container during turn and carry operations by constraining and adjusting the movements of the two rovers.

Note in Fig. 3 that we distinguish between some of the behaviors as belonging to the leader and some to the follower. This is due to the fact that the rovers have heterogeneous capabilities; the lead rover (SRR) has color stereo cameras on an actuated mast that can be used for target detection and tracking (see Fig. 4). This capability is used by the face target behavior 


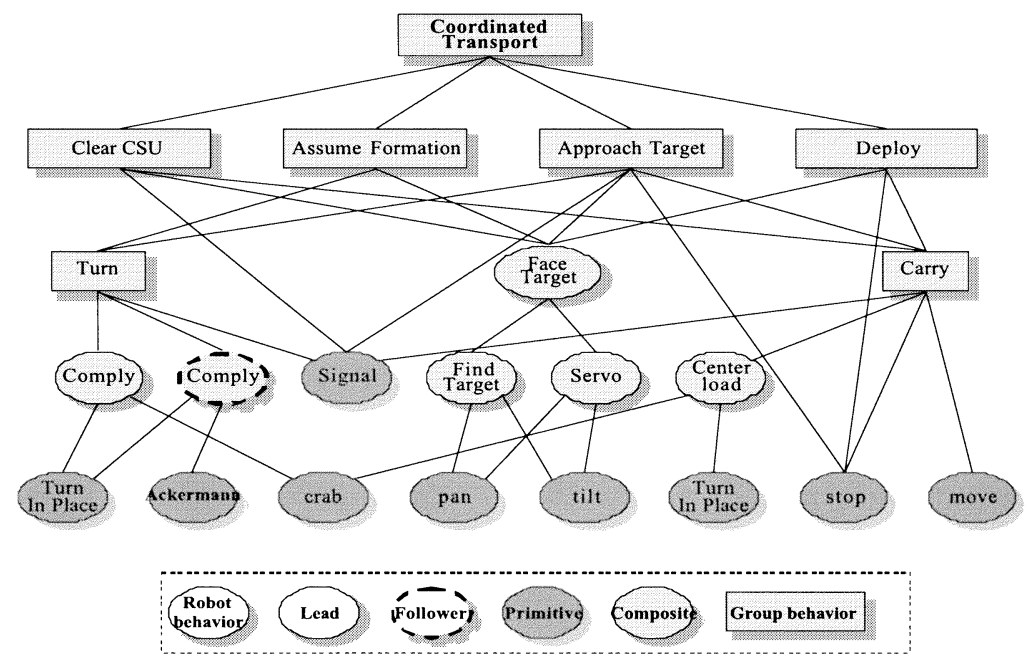

Fig. 3. Behavior hierarchy describing the coordinated transport task. Bubbles represent single robot behaviors and boxes represent group behaviors. The hierarchy shows how the behaviors are composed from lower-level behaviors.

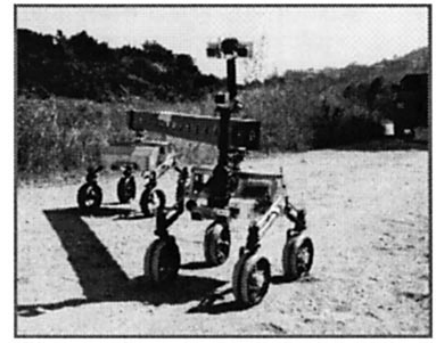

(a)

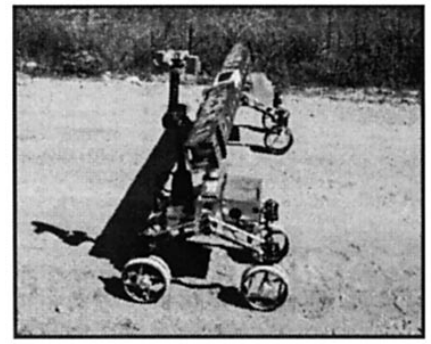

(b)
Fig. 4. Transport formations for the robot work crew (SRR in foreground and SRR2K in background). (a) Column formation for transport and obstacle avoidance. (b) Row formation for precision placement.

to align the robots to the deployment area. The follower rover (SRR2K) does not posses this capability and can not achieve the goal of facing the target on its own, instead using shadow behaviors to retrieve target information from SRR. In the current implementation, the roles of the rovers are preassigned as opposed to being dynamically determined through some negotiation or task allocation mechanism.

The assume formation group behavior is invoked to configure the two robots into a given formation, defined by the relative angle between them and the relative angle toward the target. The face target behavior provides the angle to the target and the turn group behavior reconfigures the formation to a desired one. Two constraints make this a challenging task. First, transformation between the current and target formations must ensure that the container is handled safely, i.e., the distance between the robots should always remain within some tolerance margin (typically about $1 \mathrm{~cm}$ in our setup). A set of compliance behaviors monitor the state of the load and constrain the movement of the rovers to guarantee this requirement. Second, it is required that the container does not collide with the mast on the lead rover (see Fig. 4), which could lead to damaging the mast, the gripper/gimbal or the container, and/or dropping the container. This constraint is satisfied by group movement that alternates between the lead rover turning to minimize the difference between its current and desired headings subject to a constraining safety zone about the mast, followed by the other rover pivoting to minimize the error in the formation angle.

The approach target group behavior's objective is to safely carry the container toward the deployment area. It is composed of two main group behaviors carry and turn (see Fig. 3). The main challenge of this behavior is to prevent the container from falling, which is achieved by active compliance. The compliance behaviors consist of center load and comply composite behaviors, which must comply to any external and internal disturbances caused by the rovers or the uneven terrain.

The center load behavior is activated when the force in the gripper/gimbal on either of the rovers exceeds a specified threshold. The corrective procedure is for each rover to center the load with respect to the center of its gripper/gimbal. The misalignment is illustrated by the arrows in Fig. 5. In the corrective procedure, the lead rover performs its correction while the follow rover waits. When the lead rover has completed its correction, the rovers reverse roles and the follow rover performs its correction. This is done with the four step procedure of synchronization to halt both rovers, alignment of the lead rover along the axis of the payload, driving by the lead rover along this axis, and finally the same axis alignment and driving by the follow rover.

At the lowest level, the comply behavior performs coordinated turns and straight-line formation motion of the rover pair with minimal explicit communication between the rovers. Utilizing the gripper/gimbal sensory information and the known physical constraint between the rovers imposed by the shared payload container, each rover can partially estimate its physical relationship with respect to the other rover. Using this information and knowing its role in achieving the current goal (turn or move in formation in a straight line), each rover can operate independently until the terminal condition indicating goal achievement or an exception condition occurs.

In coordinated formation driving, the rovers attempt to drive in a straight line. Each rover attempts to maintain its orientation with respect to the container (and so its orientation with respect to the other rover) using local sensory data from its gimbal. 


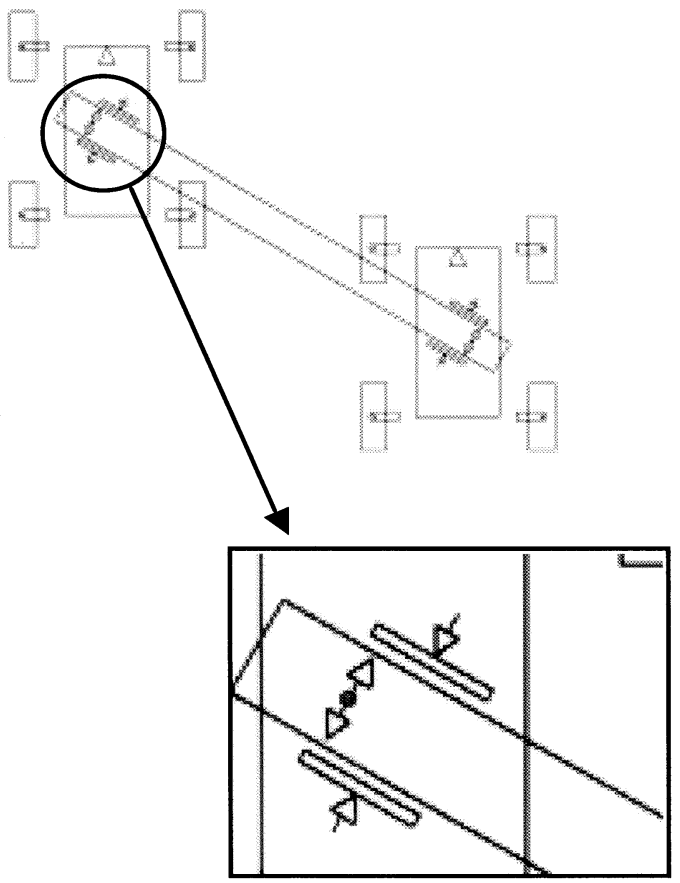

Fig. 5. Misalignment of payload in gripper/gimbal prior to invocation of center load behavior. The ideal alignment would have all of the arrows in line.

Depending on the formation (column, row or something in between), each rover uses its speed and its heading to compensate for deviations from the formation and for force build-up (compression or extension) of the container. The terminal condition for this activity is the achievement of the distance traversed as determined by the approach target group behavior (see Fig. 3). The center load and approach target group behaviors guarantee that the team will arrive at the predicted world position with a known configuration despite environmental factors such as imperfect sensors, wheel slippage, and other factors. Thresholds on force and formation angle error trigger exceptions that abort the activity.

The PD controllers defined for the comply behavior for each agent in the team independently achieve their respective goals but when implemented simultaneously will result in conflicting speed and heading corrections. To resolve these conflicts, we combined the outputs of each of the PD controllers into a single function using a weighting scheme to compute the desired speed and heading corrections for each rover. Further details can be found in [53].

\section{EXPERIMENTAL STUDIES}

We have selected a PV tent deployment scenario as our first experimental test-bed for CAMPOUT (see Fig. 6). A study was done by Colozza on the viability of a PV tent array for the power needs of a human habitat on Mars [7]. The individual containers of the PV tent elements are $5 \mathrm{~m}$ in length, so it would be difficult for a single mobile platform to manipulate and transport one to a deployment site. A four phase process for the deployment of a single PV tent by two rovers is shown in Fig. 6 . The four phases are as follows:

1) clear the CSU in preparation for a turn;

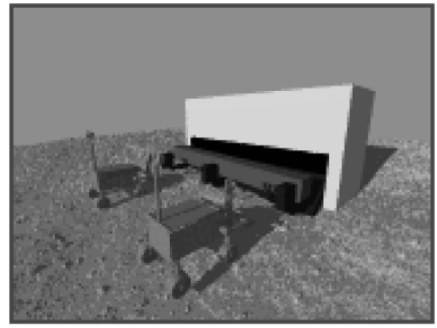

(a)

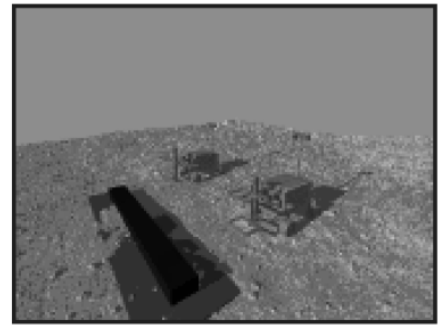

(c)

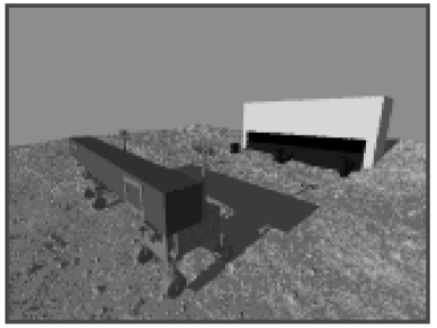

(b)

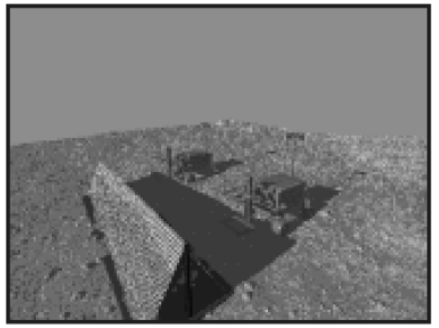

(d)
Fig. 6. Four step sequence for a PV tent array deployment. (a) Unload container from container storage unit (CSU). (b) Traverse to the deployment site; (c) Position and open the container. (d) Deploy the PV tent. PV tent storage container is $5 \mathrm{~m}$ in length and is not well handled by a single robot.

2) traverse to a staging area;

3) survey the deployment area for a clear site;

4) traverse to docking site.

The two main behaviors required for these four phases are assume transport formation, a group behavior that autonomously guides the two rovers into a specific formation such as row (side-by-side) or column (leader-follower) as shown in Fig. 4, and coordinated transport, a group behavior that autonomously controls the system during any traversal.

Our studies, the past two years, have concentrated on Steps 1 and 2, the unloading and traverse to the deployment site [shown in Fig. 1(d)] [31], [53]. The tightly coupled multirobot system depicted in Fig. 4 is composed of two rovers with independent all-wheel drive and steering. The two rovers are mechanically coupled through a $2.5 \mathrm{~m}$ long hollow beam with a $0.25 \mathrm{~m}$ by $0.25 \mathrm{~m}$ square cross-section (half-size mockup of a PV tent). This payload is held at its ends by the rovers with four degree-of-freedom (DOF) passive compliant gimbals (see Fig. 7). The gimbals are mounted to the top of the rovers at a point along the centerline of their turn-in-place rotation. This allows each rover to turn in place under ideal conditions without affecting the payload position. The four potentiometer-instrumented DOF of the gimbals are pitch, yaw, roll, and sliding along the longitudinal axis of the payload. The pitch and yaw DOF have springs that return the gimbals to the vertical positions. The slider allows translation of the beam within its grippers of plus or minus 0.02 meters. Force and torque in the gimbals can also be sensed using a 6 DOF force/torque sensor mounted at the base of the gimbals.

The average results of the first experimental studies with ten runs of the system using the control structure shown in Fig. 3 demonstrated

1) 40-50 meter autonomous traverses of outdoor irregular terrain (maximal slope of $9^{\circ}$ ) by two rovers 


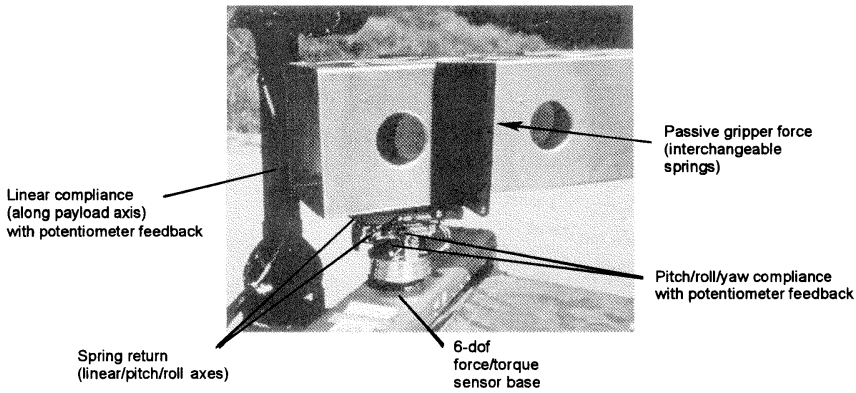

Fig. 7. Compliant gimbal instrumented with position, angle, and force feedback sensors used to hold and sense the container.

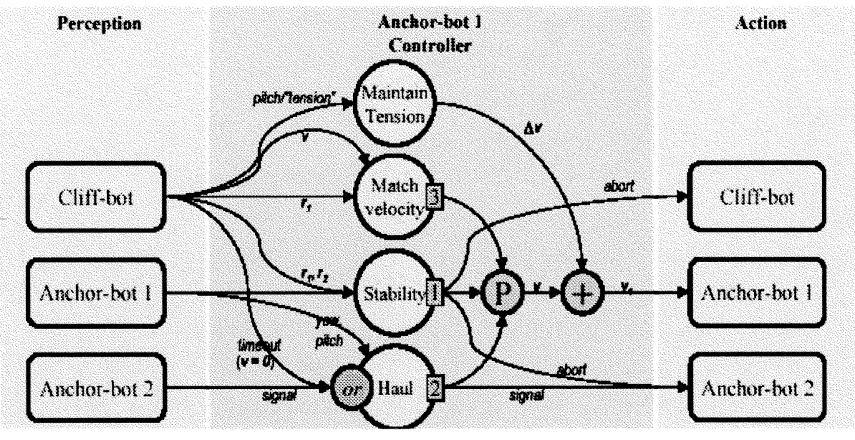

Fig. 8. A subset of the behavior network for collective cliff-descent illustrating the sub-system for controlling the velocity of anchor-botl. The arrows represent data links between local blocks as well as remote components (behaviors, sensors, actuators) thus spanning a behavior network across the team of robots.

(SRR/SRR2k) in the tightly coupled transport of an extended container:

2) autonomous approach to the CSU and coordinated grasping of the payload container in outdoor irregular terrain (maximal slope of $2^{\circ}$ ) by two rovers (SRR/SRR2K);

3) autonomous change of formation by two rovers carrying an extended container under compliant control;

4) continuous, autonomous visual guidance to a designated deployment site from $50 \mathrm{~m}$, with a heading error $<1^{\circ}$; and a distance error $<5 \%$ by use of a visual template.

Our second experimental study used CAMPOUT to build a distributed behavior network with an active agent (cliff-bot) traversing a cliff face through collective way point navigation while being stabilized with tethers from two other active agents (anchor-bots) at the top of the cliff [shown in Fig. 1(b)] [54]. The behavior network for one of the anchored agents is shown in Fig. 8. The four main behaviors in the network are maintain tension used to keep a constant tension on the tethers, match velocity used to coordinate the velocity of the active agent on the cliff face with the tether playout, stability used to minimize the possibility of tip-over by the active agent, and haul used to put tension on the tethers if the active agent doesn't have enough torque when it first starts to move. These behaviors are fused using priority weighting, with stability having the highest priority.

In theory, the coordination of the velocities of anchor-bots with the cliff-bot boils down to a straight-forward projection of velocities onto the tether vectors. However, there are a number of failure modes that are not handled by this approach. These include the possible lack of torque mode, where the velocity of the cliff-bot is zero, and would remain zero unless otherwise assisted. CAMPOUT facilitates distributed control and estimation through access to data that is distributed across the robots in the shadow behaviors (relative positions of robots can be inferred due to tether configuration).

Ten successful experiments were conducted on the mesa north of JPL on natural, challenging hill-sides [see Fig. 1(b)]. The experiments demonstrated way-point navigation to designated science targets on slopes $>70^{\circ}$ over distances of 10-15 meters (distance as limited by physical site access restrictions). The "anchor-bots" worked under a collective estimation and distributed control with the descending "cliff-bot" to enable a robust, fault-free traverse in arbitrary directions.

\section{CONCLUSION}

We have presented a control architecture called CAMPOUT for the system level coordination of multiple mobile robots. The design is three-layer, with a behavior-based middle layer. The lowest level in CAMPOUT is built using legacy device drivers from previous rover tasks such as SRR and FIDO [3]-[5]. In our two experimental testbed scenarios of autonomous robotic grasping and transport of extended payloads over irregular natural terrain and way point navigation on steeply sloped cliff faces, the capabilities of CAMPOUT for distributed multiagent control were successfully demonstrated. CAMPOUT enabled autonomous traversals of up to 50 meters over uneven terrain by a team of two rovers transporting an extended payload using nonactuated grippers. It also enabled a team of three rovers (two anchored, one active) to traverse to given waypoints on cliff faces of up to $70^{\circ}$ slopes. Based on these experimental studies, CAMPOUT has demonstrated the necessary capabilities of autonomous, distributed control that are key to the next generation of NASA missions, including multiagent inspection and maintenance of the International Space Station and the L2 next generation telescope. During the next fiscal year we will concentrate on the addition of planning and scheduling capabilities into CAMPOUT and further field experimentation.

\section{REFERENCES}

[1] R. Colonies, Autonomous Robots, R. Arkin and G. Bekey, Eds. Norwell, MA: Kluwer, 1997.

[2] P. S. Schenker, E. T. Baumgartner, S. Lee, H. Aghazarian, M. S. Garrett, R. A. Lindemann, D. K. Brown, Y. Bar-Cohen, S. S. Lih, B. Joffe, S. S. Kim, B. H. Hoffman, and T. L. Huntsberger, "Dexterous robotic sampling for Mars in-situ science," in Proc. SPIE Conf. Intelligent Robotics Computer Vision XVI, vol. 3208, Pittsburgh, PA, Oct. 14-17, 1997.

[3] P. S. Schenker, E. T. Baumgartner, R. A. Lindemann, H. Aghazarian, D. Q. Zhu, A. J. Ganino, L. F. Sword, M. S. Garrett, B. A. Kennedy, G. S. Hickey, A. S. Lai, L. H. Matthies, B. D. Hoffman, and T. L. Huntsberger, "New planetary rovers for long range mars science and sample return," in Proc. SPIE Conf. Intelligent Robotics Computer Vision XVII, vol. 3522, Boston, MA, Nov 1-5, 1998.

[4] T. L. Huntsberger, E. T. Baumgartner, H. Aghazarian, Y. Cheng, P. S. Schenker, P. C. Leger, K. D. Iagnemma, and S. Dubowsky, "Sensorfused autonomous guidance of a mobile robot and applications to Mars sample return operations," in Proc. SPIE Conf. Sensor Fusion Decentralized Control Robotic Systems II, vol. 3839, Boston, MA, Sept. 19-22, 1999, pp. 2-8. 
[5] C. R. Weisbin, G. Rodriguez, P. S. Schenker, E. Baumgartner, R. Volpe S. Hayati, and H. Das, "Autonomous rover technology for Mars sample return," in Proc. 5th Int. Symposium on Artificial Intelligence, Robotics and Automation in Space, Noordwijk, The Netherlands, June 1-3, 1999.

[6] R. Volpe, E. Baumgartner, P. Schenker, and S. Hayati, "Technology development and testing for enhanced Mars rover sample return operations," in Proc. IEEE Aerospace 2000, Big Sky, MT, Mar. 2000, pp. 246-257.

[7] A. Colozza, "Design and Optimization of a Self-Deploying PV Tent Array," Lewis Research Center Group, Brook Park, OH, NASA CR187 119, 1991.

[8] S. I. Roumeliotis and G. A. Bekey, "Collective localization: A distributed kalman filter approach to localization of groups of mobile robots," in Proc. IEEE Int. Conf. Robotics Automation, San Francisco, CA, Apr. 22-28, 2000, pp. 2958-2965.

[9] D. Fox, W. Burgard, H. Kruppa, and S. Thrun, "A probabilistic approach to collaborative multi-robot localization," Auton. Robots, vol. 8, no. 3, 2000.

[10] B. B. Werger and M. J. Mataric, "Robotic "food" chains: Externalization of state and program for minimal-agent foraging," in Proc. From Animals to Animats 4, 4th Int. Conf. Simulation Adaptive Behavior, P. Maes, M. Mataric, J. Meyer, J. Pollack, and S. W. Wilson, Eds., 1996, pp. 625-634.

[11] R. C. Arkin, "Cooperation without communication: Multi-agent schema based robot navigation," J. Robot. Syst., vol. 9, no. 3, pp. 351-364, 1992.

[12] D. Goldberg and M. J. Mataric, "Coordinating mobile robot group behavior using a model of interaction dynamics," in Proc. Autonomous Agents, Seattle, WA, May 1-3, 1999, pp. 100-107.

[13] M. J. Mataric, "Behavior-based control: Examples from navigation, learning, and group behavior," J. Exper. Theoret. Artif. Intell., vol. 9, no. 2-3, pp. 323-336, 1997.

[14] D. Rus, B. Donald, and J. Jennings, "Moving furniture with teams of autonomoujs robots," in Proc. IEEE/RSJ Int. Conf. Intelligent Robots Systems, 1995, pp. 235-2442.

[15] B. Gerkey and M. J. Mataric, "Principled communication for dynamic multi-robot task allocation," in Proc. International Symposium Experimental Robotics, Waikiki, HI, Dec. 10-13, 2000.

[16] L. E. Parker, "ALLIANCE: An architecture for fault toleran multi-roibot cooperation," IEEE Trans. Robot. Automat., vol. 14, pp. 220-240, 1998.

[17] O. Khatib, K. Yokoi, K. Chang, D. Ruspini, R. Holberg, and A. Casal, "Decentralized cooperation between multiple manipulators," in Proc. 5th IEEE Int. Workshop Robot Human Communication, 1996.

[18] O. Khatib, K. Yokoi, K. Chang, D. Ruspini, R. Holmberg, and A. Casal, "Vehicle/arm coordination and multiple mobile manipulator decentralized cooperation," in Proc. IEEE/RSJ Int. Conf. Intelligent Robots Systems, 1996, pp. 546-553.

[19] A. Agah and G. A. Bekey, "In a team of robots the loudest is not necessary the best," in Proc. IEEE Int. Conf. Systems, Man, Cybernetics, 1995, pp. 3800-3805.

[20] J. Borenstein, "Control and kinematic design of multi-degree-of-freedom mobile robots with compliant linkage," IEEE Trans. Robot. Automat., vol. 11, pp. 21-35, Feb. 1995.

[21] M. Hara, M. Fukuda, H. Nishibashi, Y. Aiyama, Y. J. Ota, and T. Arai, "Motion control of cooperative transportation system by quadruped robots based on vibration model walking," in Proc. IEEE/RSJ Int. Conf. Intelligent Robots Systems, 1999, pp. 1651-1656.

[22] H. Osumi, M. Terasawa, and H. Nojiri, "Cooperative control of multiple mobile manipulators on uneven ground," in Proc. IEEE Int. Conf. Robotics and Automation, Leuven, Belgium, 1998, pp. 3198-3203.

[23] H. Hoshino, E. Muro, T. Fukuda, and Y. Adachi, "Cooperative motion control of six-legged walking robot equipped with a manipulator," in Proc. IEEE 8th Int. Conf. Advanced Robotics, 1997, pp. 77-82.

[24] H. Kimuraand and H. Katano, "Vision based motion recognition of the hexapod for autonomous assistance," in Proc. IEEE/RSJ Int. Conf. Intelligent Robots Systems, 1998, pp. 1-6.

[25] H. Kimura and G. Kajiura, "Motion recognition based cooperation between human operating robot and autonomous assistant robot," in Proc.IEEE Int. Conf. Robotics and Automation, 1997, pp. 297-303.

[26] D. Kurabayashi, J. Sasaki, and Y. Aiyama, "Cooperative transport with regrasping of torque-limited mobile robots," in Proc. IEEE/RSJ Int. Conf. Intelligent Robots and Systems, 1996, pp. 304-309.

[27] N. Miyata, J. Ota, Y. Aiyama, J. Sasaki, and T. Arai, "Cooperative transportation system with regrasping car-like mobile robots," in Proc. IEEE/RSJ Int. Conf. Intelligent Robots Systems, 1997, pp. 1754-1761.

[28] T. Sugar and V. Kumar, "Multiple cooperating mobile manipulators," in Proc. IEEE Int. Conf. Robotics and Automation, 1999, pp. 1538-1543.
[29] T. Viney, B. Postma, and T. Kangsanant, "Dynamic modeling and feedback control of a mobile robot pair cooperatively transporting a long object," in Proc. 3rd Biennial Engineering Mathematics Applications Conf. , Adelaide, Australia, July 13-16 1998.

[30] P. Pirjanian, T. Huntsberger, A. Trebi-Ollennu, H. Aghazarian, H. Das, S. S. Joshi, and P. S. Schenker, "CAMPOUT: A control architecture for multirobot planetary outposts," in Proc. SPIE Conf. Sensor Fusion and Decentralized Control in Robotic Systems III, Boston, MA, Nov 2000, pp. $221-230$.

[31] P. Pirjanian, T. L. Huntsberger, and P. S. Schenker, "Development of CAMPOUT and its further applications to planetary rover operations: A multirobot control architecture," in Proc. SPIE on Sensor Fusion and Decentralized Control in Robotic Systems IV, Newton, MA, Oct 2001.

[32] P. Pirjanian, "Behavior coordination mechanisms: State-of-the-art," Institute Robotics Intelligent Systems, Sch. Engineering, Los Angeles, Univ. Southern California, Tech. Rep. IRIS-99-375, 1999.

[33] R. A. Brooks, "A robust layered control system for a mobile robot," IEEE J. Robot. Automat., vol. 2, no. 1, pp. 14-23, 1986.

[34] J. Kosecka and R. Bajcsy, "Discrete event systems for autonomous mobile agents," in Proc. Intelligent Robotic Systems, Zakopane, July 1993 , pp. 21-31

[35] J. Rosenblatt, "The distributed architecture for mobile navigation," $J$. Exper. Theoret. Artif. Intell., vol. 9, no. 2/3, pp. 339-360, 1997.

[36] R. C. Arkin, "Motor schema based navigation for a mobile robot: An approach to programming by behavior," in Proc. IEEE Int. Conf. Robotics Automation, 1987, pp. 264-271.

[37] A. Saffiotti, K. Konolige, and E.-H. Ruspini, "A multivalued logic approach to integrating planning and control," Artif. Intell., vol. 76, pp. 481-526, Mar. 1995.

[38] J. Yen and N. Pfluger, "A fuzzy logic based extension to Payton and Rosenblatt's command fusion method for mobile robot navigation," IEEE Trans. Syst., Man, Cybern., vol. 25, pp. 971-978, 1995.

[39] P. Pirjanian, "Multiple objective behavior-based control," J. Robot. Auton. Syst., vol. 31, no. 1-2, pp. 53-60, 2000.

[40] P. Pirjanian and M. Mataric, "Multiple objective vs. fuzzy behavior coordination," in Lecture Notes Computer Science Fuzzy Logic Techniques Autonomous Vehicle Navigation, D. Drainkov and A. Saffiotti, Eds., 2001.

[41] E. Gat, "Three-layer architectures," in Artificial Intelligence and Mobile Robots, D. Kortenkamp, R. P. Bonasso, and R. Murphy, Eds. Cambridge, MA: MIT Press, 1998.

[42] T. Huntsberger, M. Mataric, and P. Pirjanian, "Action selection within the context of a robotic colony," in Proc. SPIE Sensor Fusion Decentralized Control Robotic Systems II, vol. 3839, Boston, MA, 1999.

[43] T. L. Huntsberger, G. Rodriguez, and P. S. Schenker, "Robotics challenges for robotic and human mars exploration," in Proc. ROBOTICS Albuquerque, NM, Mar. 2000.

[44] T. L. Huntsberger, H. Aghazarian, E. Baumgartner, and P. S. Schenker, "Behavior-based control systems for planetary autonomous robot outposts," in Proc. IEEE Aerospace, Big Sky, MT, Mar. 2000, pp. 679-686.

[45] L. E. Parker, "Current state of the art in distributed robot systems," in Distributed Autonomous Robotic Systems 4, L. E. Parker, G. Bekey, and J. Barhen, Eds. New York: Springer-Verlag, 2000, pp. 3-12.

[46] Y. Cao, A. S. Fukunaga, and A. B. Kahng, "Cooperative mobile robotics: Antecedents and directions," Auton. Robot., vol. 4, no. 1, pp. 7-27, 1997.

[47] T. L. Huntsberger and J. Rose, "BISMARC," Neural Netw., vol. 11, no. 7/8, pp. 1497-1510, 1998.

[48] P. Caloud, W. Choi, J.-C. Latombe, L. C. Pape, and M. Yin, "Indoor automation with many mobile robots," in Proc. IEEE/RSJ IROS, 1990, pp. 67-72.

[49] H. Asama, A. Matsumoto, and Y. Ishida, "Design of an autonomous and distributed robot system: ACTRESS," in Proc. IEEE/RSJ IROS, 1989, pp. 283-290.

[50] K. Jin, P. Liang, and G. Beni, "Stability of synchronized control of discrete swarm structures," in Proc. IEEE Int. Conf. Robotics Automation, 1994, pp. 1033-1038.

[51] R. Simmons, S. Singh, D. Hershberger, J. Ramos, and M. F. Smith III, "First results in the coordination of heterogeneous robots for large-scale assembly," in Proc. ISER 7th Int. Symp. Experimental Robotics, Dec. 2000.

[52] P. Pirjanian and M. Mataric, "Multi-robot target acquisition using multiple objective behavior coordination," in Proc. IEEE Int. Conf. Robotics Automation, San Francisco, CA, Apr. 2000, pp. 101-106.

[53] A. Trebi-Ollennu, H. Das, H. Aghazarian, A. Ganino, P. Pirjanian, T. Huntsberger, and P. Schenker, "Mars rover pair cooperatively transporting a long payload," in Proc. IEEE Int. Conf. Robotics Automation, Washington, DC, May 2002, pp. 3136-3141. 
[54] P. Pirjanian, C. Leger, E. Mumm, B. Kennedy, M. Garrett, H. Aghazarian, P. S. Schenker, and S. Farritor, "Distributed control for a modular, reconfigurable cliff robot," in Proc. IEEE Int. Conf. Robotics Automation, Washington, DC, May 2002, pp. 3136-3141.

[55] D. Apostolopoulos and J. Bares, "Locomotion configuration of a robust rappelling robot," in Proc. IEEE/RSJ Int. Conf. Intelligent Robots Systems, vol. 3, Aug. 1995, pp. 280-284.

[56] B. B. Werger, "AYLLU: Distributed port-arbitrated behavior-based control," in Distributed Autonomous Robotic Systems 4, L. E. Parker, G. Bekey, and J. Barhen, Eds. New York: Springer-Verlag, 2000, pp. 25-34.

[57] L. Chaimowicz, T. Sugar, V. Kumar, and M. F. M. Campos, "An architecture for tightly coupled multi-robot cooperation," in Proc. IEEE Int. Conf. Robotics Automation, Seoul, Korea, May 2001, pp. 2992-2997.

[58] T. L. Huntsberger, "Biologically inspired autonomous rover control," Auton. Robot., vol. 11, no. 11, pp. 341-346.

[59] D. Lyons and A. Hendriks, "Planning as incremental adaptation of a reactive system," Robot. Autonom. Syst., vol. 14, no. 4, pp. 255-288, 1995.

[60] R. C. Arkin, Behavior-Based Robotics. Cambridge, MA: MIT Press, 1998.

[61] C. R. Kube and H. Zhang, "Task modeling in collective robotics," Auton. Robots, vol. 4, no. 1, pp. 53-72, 1997.

[62] Z. D. Wang, E. Nakano, and T. Takahashi, "Solving function distribution and behavior design problem for cooperative object handling by multiple mobile robots," IEEE Trans. Syst., Man, Cybern. B, 2004, to be published.

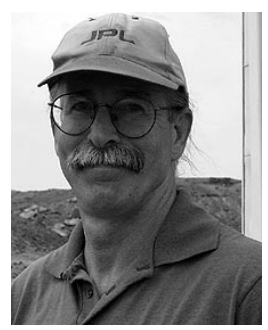

Terry Huntsberger received the Ph.D. degree in physics from the University of South Carolina, Columbia, in 1978.

He is a Senior Member of the Technical Staff in the Mechanical and Robotics Technologies Group at the Jet Propulsion Laboratory, Pasadena, CA, where he is the Manager for numerous tasks in the areas of multirobot control systems, rover systems for access to high-risk terrain, and long range traverse algorithms for rovers. He is a member of the planning teams for the Mars Science Laboratory (MSL) mission planned for launch in 2009. He is an Adjunct Professor and former Director of the Intelligent Systems Laboratory in the Department of Computer Science at the University of South Carolina. His research interests include behavior-based control, computer vision, neural networks, wavelets, and biologically inspired system design. He has published over 120 technical articles in these and associated areas.

Dr. Huntsberger is a member of SPIE, ACM, and INNS.

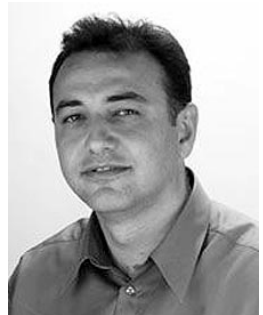

Paolo Pirjanian received the M.Sc.CS. and the Ph.D. degrees from Aalborg University, Aalborg, Denmark, in 1994 and 1998, respectively

He was formerly a Member of the Engineering Staff in the Mechanical and Robotics Technologies Group at the Jet Propulsion Laboratorym (JPL), Pasadena, $\mathrm{Ca}$, and is currently the Chief Scientist for Evolution Robotics, Pasadena, CA. During his tenure at JPL, he was the System Engineer for the Reconfigurable Robotic Surface Systems/All Terrain Explorer and a System Architect for the Robot Work Crews tasks. He was the primary developer of CAMPOUT, and is working toward the development of new, sustainable, and aggressive mobility systems for autonomous access to highly variable terrain, cliffs, etc. using state-of-the-art multifunctional, modular, and reconfigurable sensing, controls, hardware, and robotic systems. Until December 1998, he held an Assistant Research Professor position at the Laboratory of Image Analysis, Aalborg University. From January 1999 to November 1999, he was a Postdoctoral Research Associate at the USC Robotics Research laboratory working on multiple robot coordination with Maja Mataric. He has over 30 technical publications in the fields of robotics, control, and computer vision. He serves as a part-time Lecturer at the Computer Science Department of University of Southern California, Los Angeles.

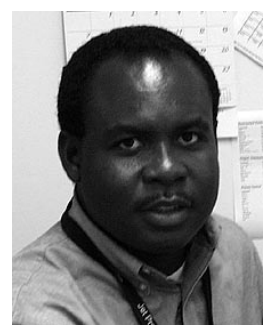

Ashitey Trebi-Ollennu received the B.Eng. degree (Hons.) in avionics engineering from Queen Mary College, University of London, U.K., and the Ph.D. degree in aerospace engineering at the Royal Military College of Science, Cranfield University, U.K., in 1991 and 1996, respectively

$\mathrm{He}$ is a Member of Engineering Staff at the Jet Proportion Laboratory in Pasadena, CA, where he serves as a Control Systems, Operations and Test Engineer for the Field Integrated Design and Operations Rover (FIDO), Robot Work Crews and Mars Exploration Rover (MER) 2003 projects. He is a multidisciplinary engineer with a broad background and extensive experience in planetary rover operations, distributed mobile robotics, system architectures, dynamic modeling and control systems design for aerospace and mechatronics systems. His current research at JPL focuses on planetary rovers, multiple mobile robots (planetary outpost), reconfigurable robots and man-machine interaction. His research has resulted in more than 50 journal articles, conference papers, and book chapters. He was a Research Scholar at, Institute for Complex Engineered Systems, Carnegie Mellon University, Pittsburgh, PA, were he helped develop a system of All Terrain Vehicles (ATVs) for distributed tactical surveillance for DARPA.

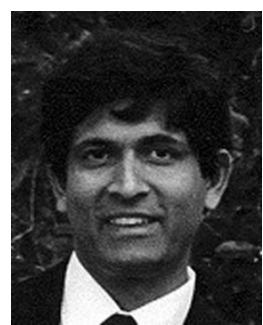

Hari Das Nayar received the B.S., M.S., and Sc.D. degrees in mechanical engineering, Massachusetts Institute of Technology, Cambridge, in 1984 and 1989 , repectively.

He worked at Systemantics, Inc. from 1989 to 1990 and at the Jet Propulsion Laboratory, California Institute of Technology, Pasadena, CA, from 1990 to 2002. He was a Visiting Lecturer at the University of California at Los Angeles. He also founded OphirTech in 2002 to develop and commercialize robotics, engineering analysis, and educational

products

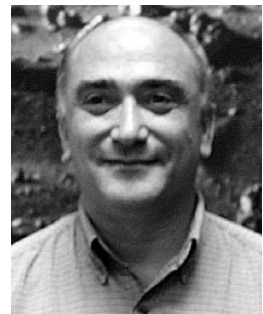

Hrand Aghazarian received the dual B.S. degree in applied math and computer science, and the M.S. degree in computer science from California State University, Northridge, in 1987 and 1990, respectively.

$\mathrm{He}$ is a Senior Member of the Engineering Staff in the Mechanical sand Robotics Technologies Group at the Jet Propulsion Laboratory, Pasadena, CA. He has extensive work experience in the design and development of real-time embedded software for a variety of avionics systems. Currently he is involved in providing software solutions in the area of software architecture, low-level drivers, motor control, user interfaces for commanding planetary rovers, and navigation algorithm for SRR and FIDO rovers. His research interests are rover navigation/control and real-time embedded software design and development.

Mr. Aghazarian is a member of ACM and INNS.

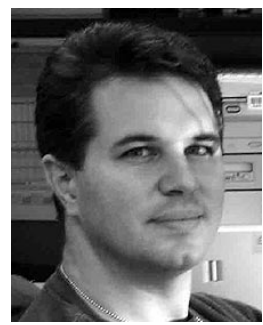

Anthony J. Ganino received the B.S. and M.S. degrees in mechanical engineering from Virginia Polytechnic Institute and State University, Blacksburg, in 1994 and 1996, respectively.

$\mathrm{He}$ is a Member of the Engineering Staff at the Jet Propulsion Laboratory in Pasadena, CA, where he has been involved in the design, manufacture, and integration of both research-based and flight robotic systems. He has actively participated in, or led, the mechanical design efforts of a number of robotic vehicle projects including: lightweight survivable rover (LSR), sample return rover (SRR), robotic work crews, all-terrain explorer, tactical mobile robots, and the field integrated development and operations (FIDO) rover. Most recently, he has been responsible for various aspects of the integration and assembly of the two Mars exploration rover flight systems as a member of the assembly test and launch operations (ATLO) engineering team. 


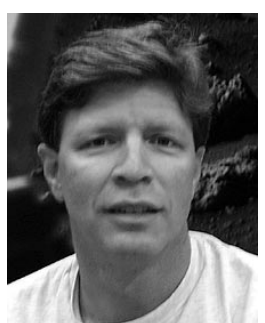

Mike Garrett (M'01-A'01) received the B.S and M.S. degrees in mechanical engineering from Virginia Polytechnic Institute and State University, Blacksburg, in 1994 and 1996, respectively.

$\mathrm{He}$ is a Member of the Engineering Staff at the Jet Propulsion Laboratory, Pasadena, CA. He has designed electronics and sensors for multiple JPL research rovers including SRR and FIDO and for MER flight systems ground support equipment. He has worked on various research and flight projects since 1980.

Mr. Garrett is a member of ISA.

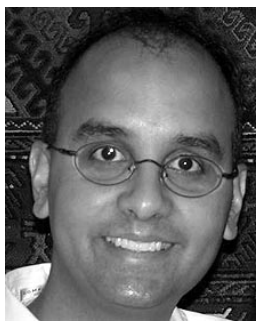

Sanjay S. Joshi was formerly a Senior Member of the Engineering Staff at the Jet Propulsion Laboratory, Pasadena, CA, and is currently an Assistant Professor in the Department of Mechanical and Aeronautical Engineering at the University of California, Davis. His research interests include autonomous systems, robotics, and control systems.

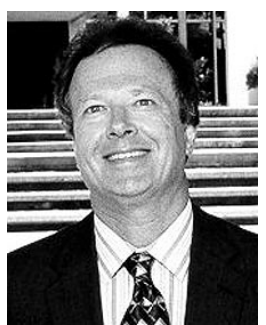

Paul S. Schenker (M'76) is Manager, Mobility Systems Concepts Development Section, Jet Propulsion Laboratory (JPL), California Institute of Technology, Pasadena, and as such, is responsible for JPL R\&D in planetary mobility and robotics. His published research includes topics in robotic perception, robo control architectures, telerobotics and teleoperation, multisensor fusion, and most recently, multirobot cooperation. He has led the development of robotic systems that include the field integrated design and operations rover (FIDO), planetary dexterous manipulator (MarsArm, microArm), robot assisted microsurgery system (RAMS), robotic work crew (RWC), and all-terrain explorer (ATE/Cliff-bot), with resulting technology contributions to NASA missions.

Dr. Schenker is an active member of Optical Society of America and SPIE He has served as an elected board member and 1999 President and currently serves as an elected member of the NAS/United States Advisory Committee to the International Commission for Optics. 